\section{AVALIAÇÃO DA VIGILÂNCIA EPIDEMIOLÓGICA AMPLIADA DO ROTAVÍRUS}

\author{
Evaluation of rotavirus enhanced epidemiologic surveillance \\ Evaluación de la vigilancia epidemiológica ampliada del \\ rotavirus
}

\section{RESUMO}

Objetivo: Conhecer as práticas de vigilância do Rotavírus, identificando os pontos positivos e as dificuldades encontradas através do modelo de avaliação da Vigilância Epidemiológica Ampliada das Doenças Diarreicas por Rotavírus (VER). Métodos: Tratou-se de uma pesquisa avaliativa realizada em dois diferentes estados brasileiros, entre março a novembro de 2010. Participaram da avaliação 14 atores no total (do nível central, estadual e municipal), sendo submetidos a entrevistas gravadas utilizando questionários semiestruturados, além da realização de análise documental e observação direta da rotina do serviço dentro das respectivas unidades. Essas informações permitiram alimentar uma matriz de julgamento e evidenciar os variados aspectos componentes da operacionalização do sistema. Resultados: Foram observados pontos positivos (a sensibilidade de captação de casos, a aceitabilidade do sistema por parte dos técnicos envolvidos, a estrutura física adequada e a capacitação e atualização dos técnicos) e dificuldades operacionais (a escassez de recursos humanos e financeiros, e a baixa oportunidade do sistema de informação) na condução das atividades da vigilância. Conclusão: Os resultados encontrados sugerem a baixa efetividade do sistema de vigilância adotado, indicando que a vigilância do tipo sentinela pode não ser a mais adequada para o sistema de saúde desses estados estudados.

Descritores: Vigilância Epidemiológica; Rotavírus; Avaliação em Saúde; Diarreia.

\section{ABSTRACT}

Objective: To assess the practices of Rotavirus surveillance, identifying the positive aspects and the difficulties found through the evaluation model from the Enhanced Surveillance of Rotavirus Diarrheal Diseases. Method: This is an evaluative and qualitative research performed in two different Brazilian states between March and November 2010. Participated in the evaluation 14 subjects in total (from central, state and municipal level), which were submitted to tape-recorded interviews using semi-structured questionnaires, along with conduction of document analysis and direct observation of routine service within their respective units. This information allowed to feed a judgment matrix, thus highlighting the various aspects comprising the system operation. Results: Positive aspects were observed (the sensitivity to capture cases, the system acceptability by the technicians involved, the proper physical structure and the training and updating of technicians), and operational difficulties when conducting surveillance activities (the shortage of human and financial resources and the low opportunity of the information system). Conclusion: The results suggest the low effectiveness of the monitoring system adopted, indicating that the sentinel type of surveillance may not be the most appropriate for the health system in the studied states.

Decriptors: Epidemiological Surveillance; Rotavirus; Health Evaluation; Diarrhea.
Descrição ou avaliação de experiências, métodos, técnicas, procedimentos e instrumentais
1) Escola Nacional de Saúde Pública Sérgio Arouca - ENSP - Fundação Oswaldo Cruz Fiocruz - Rio de Janeiro (RJ) - Brasil.
Recebido em: 25/01/2013 Revisado em: 02/07/2013 Aceito em: 02/12/2013 


\section{RESUMEN}

Objetivo: Conocer las prácticas de vigilancia del Rotavirus identificando los puntos positivos y las dificultades encontradas a través del modelo de evaluación de la Vigilancia Epidemiológica Ampliada de las Enfermedades Diarreicas por Rotavirus. Métodos: Se trató de una investigación evaluativa realizada en dos estados brasileños distintos entre marzo y noviembre de 2010. En total 14 actores participaron de la evaluación (del nivel central, estadual y municipal), siendo sometidos a entrevistas grabadas utilizando cuestionarios semi-estructurados y la realización de un análisis documental y observación directa de la rutina del servicio en las respectivas unidades. Estas informaciones permitieron fomentar una matriz de juicio y evidenciar los distintos aspectos de la utilización del sistema. Resultados: Fueron observados puntos positivos (la sensibilidad de captación de casos, la aceptabilidad del sistema por parte de los técnicos involucrados, la estructura física adecuada y la captación y actualización de los técnicos) $y$ dificultades operacionales (la escasez de recursos humanos y financieros y la baja oportunidad del sistema de información) en la conducción de las actividades de vigilancia. Conclusión: Los resultados encontrados sugieren baja efectividad del sistema de vigilancia adoptado e indica que la vigilancia del tipo centinela puede que no sea la más adecuada al sistema de salud de los estados investigados.

Descriptores: Vigilancia Epidemiológica; Rotavirus; Evaluación en Salud; Diarrea.

\section{INTRODUÇÃO}

O gênero Rotavirus, pertencente à família Reoviridae, está associado à ocorrência de doença diarreica entre crianças menores de cinco anos de idade, tanto em países desenvolvidos quanto naqueles em desenvolvimento ${ }^{(1)}$.

Em todo o mundo, são notificadas anualmente 1,9 milhão de mortes por doenças diarreicas agudas, das quais, um terço estão associadas à infecção pelo Rotavírus ${ }^{(2,3)}$. Estima-se que noventa por cento dessas mortes ocorrem em países em desenvolvimento, nos quais as condições de saneamento básico, estado nutricional e acesso aos serviços de saúde são mais deficientes ${ }^{(4,5)}$.

Em países desenvolvidos, como os Estados Unidos, os prejuízos decorrentes destas infecções são estimados em aproximadamente 1 bilhão de dólares por ano ${ }^{(6)}$. No Brasil, a infecção por esse vírus representa cerca de $30 \%$ do total de casos de Doenças Diarreicas Agudas (DDA) ocorridas no país ${ }^{(7,8)}$.

Desde 2005, duas novas vacinas de vírus vivos orais atenuados foram licenciadas para uso em diversos países para o controle da rotavirose, uma monovalente e a outra pentavalente. Ambas têm sido efetivas na prevenção de formas mais graves das doenças diarreicas ${ }^{(4,9-10)}$. O Brasil foi o primeiro país da América Latina a introduzir a vacina monovalente em seu programa nacional de imunização, em março de $2006^{(11)}$.

Considerando o impacto financeiro e social dessas infecções, a Organização Pan-Americana de Saúde (OPAS) propôs, em 2003, um modelo de Vigilância Epidemiológica Ampliada das Doenças Diarreicas por Rotavírus (VER) ${ }^{(12,13)}$. O Brasil, assim como outros países das Américas, vem implantando a vigilância preconizada desde 2006, fundamentada no estabelecimento de unidades sentinelas ${ }^{(14)}$.

A VER no Brasil tem como objetivos conhecer o perfil das gastroenterites causadas por Rotavírus no país, em crianças menores de cinco anos; estimar a magnitude da doença diarreica por Rotavírus no país; apoiar a implementação de medidas necessárias para o controle; identificar os sorotipos e genótipos circulantes no país; e avaliar o impacto da vacina introduzida no calendário do Programa Nacional de Imunização para o Rotavírus (PNI/ Rotavírus) ${ }^{(4)}$.

Este modelo de vigilância sentinela, embora apresente limitações do ponto de vista de representatividade da população geral, pode produzir dados fundamentais para estudos epidemiológicos com custo reduzido. Segundo o Ministério da Saúde do Brasil, até dezembro de 2013, 17 das 27 unidades federativas do país haviam implantado a $\operatorname{VER}^{(14)}$.

Por encontrar-se em implantação e ainda possuir diversos pontos que impossibilitam seu pleno funcionamento, esse estudo teve o objetivo de conhecer as práticas de vigilância do Rotavírus, identificando os pontos positivos e as dificuldades encontradas através do modelo de avaliação da Vigilância Epidemiológica Ampliada das Doenças Diarreicas por Rotavírus (VER).

\section{MÉTODOS}

Entre março e novembro de 2010 realizou-se uma pesquisa avaliativa ${ }^{(15-17)}$ da Vigilância Epidemiológica Ampliada do Rotavírus no Brasil (VER). Em um primeiro momento, foi adaptado um modelo avaliativo a partir de publicações entre os anos de 1994 e $2010^{(18-24)}$. O modelo avaliativo envolve em sua estrutura um Modelo Lógico de Avaliação e uma matriz de julgamento.

$\mathrm{Na}$ primeira etapa desta adaptação, elaborou-se o Modelo Lógico de Avaliação (Figura 1), que pode ser entendido como um esquema visual que apresenta como o programa idealmente deve funcionar para alcançar os resultados esperados ${ }^{(24-26)}$. Desta forma, o modelo ajuda a visualizar e compreender como a estrutura do sistema (recursos humanos, físicos e financeiros) pode contribuir 
para melhorar e atingir os objetivos do $\operatorname{programa}^{(22,24-26)}$. Além disso, a utilização de modelos lógicos é uma forma eficaz de viabilizar o sucesso de programas através do planejamento, execução e implementação das avaliações.

A matriz de julgamento é um instrumento que determina os pontos necessários para que a VER seja considerada um sistema que opera de forma a promover a efetividade das ações avaliadas. Essa matriz é composta pelos seguintes componentes:

Dimensão - Para esse estudo avaliaram-se oito dimensões, sendo essas: programa e planificação, recursos financeiros e humanos, sistema de informação, estrutura, recurso logístico, supervisão e vacina. Dentro de cada dimensão, todos os níveis operacionais foram avaliados, buscando responder aos indicadores propostos;

Nivel Operacional - são os componentes estruturais que compõem a vigilância do Rotavírus, nos vários níveis operacionais, sendo: Unidades de Saúde Sentinelas (USS), Vigilância Epidemiológica Municipal (VE-SMS), Laboratórios Centrais de Saúde Pública (LACEN), Laboratórios de Referência Regional e Nacional (LRR, LRN), Secretarias Estaduais de Saúde (SES), Coordenação de Vigilância das Doenças de Transmissão Hídrica e Alimentar (COVEH/SVS), Coordenação Geral de Laboratórios de Saúde Pública (CGLAB) e Programa Nacional de Imunização para o Rotavírus (PNI/Rotavírus);

Indicadores - são variáveis, características ou atributos analisados dentro das unidades que compõem o sistema de VER. Estes indicadores, assim como seus respectivos critérios de avaliação foram elaborados a partir de diretrizes, documentos e manuais disponibilizados pelo Ministério da Saúde ${ }^{(5)}$ e pela Organização Pan-Americana de Saúde ${ }^{(12)}$. Elaboraram-se os indicadores aceitabilidade e qualidade dos dados com base na experiência descrita pelos atores entrevistados. Avaliou-se um total de 26 indicadores (Quadro I) ao final deste estudo;

Padrão de referência - são os critérios utilizados para o julgamento do indicador como adequado ou inadequado. Para alguns indicadores, não foi possível definir um valor de referência padrão, devido ao seu caráter qualitativo. Em função disso, o julgamento foi baseado em critério próprio definido pelos atores entrevistados;

Categoria - define se o padrão de referência está presente ou ausente, oportuno ou não, com boa aceitabilidade ou não, ou se com sensibilidade adequada ou não.

Em conjunto com a área técnica responsável do Ministério da Saúde pela VER, para a aplicação do modelo avaliativo, dois estados brasileiros participaram da avaliação, segundo critérios de desempenho operacional, representatividade geográfica e autonomia, sendo que sua identificação foi omitida e passaram a ser designados pelas letras A e B.

Entre abril e novembro de 2010, com a finalidade de avaliar operacionalmente a VER, entrevistaram-se 14 técnicos no total, abrangendo todos os níveis operacionais da vigilância do Rotavirus, sendo: 4 técnicos no nível federal, 1 técnico no nível estadual em cada estado e 4 técnicos em cada município participante. Nessa pesquisa, os municípios participantes foram as capitais dos estados selecionados, pois era onde as Unidades de Saúde Sentinela e secretarias municipais e estaduais estavam localizadas.

Em cada nível os atores foram submetidos a entrevistas gravadas utilizando questionários semiestruturados, que continham questões abertas de cunho discursivo e questões fechadas, referentes às varias dimensões identificadas pelo modelo lógico, como: Programa e Planificação (existência de: documentos, marco regulatório ou protocolo que oriente quanto às atribuições e condutas para as atividades; plano de ação; ocorrência de reuniões para discussões sobre metas e problemas; e mecanismos de coordenação intrainstitucional), Recurso Financiamento e Humanos (tipos de fontes financeiras; forma de repasse financeiro; disponibilidade e alocação dos recursos; existência de numero adequado de técnicos para cumprir as tarefas; existência de plano de capacitação), Sistema de Informação (existência de: documento explicitando o funcionamento do fluxo de informação e materiais; definição de caso, documentos e protocolos sobre como preencher a ficha de notificação e inserir no banco de sistema designado; e presença de feedback para as unidades notificantes), Recursos Logísticos (como é feita a aquisição do material para coleta amostras; realização de armazenamento de amostras e como essas são armazenadas; destino e envio das amostras; frequência do envio de amostras), Supervisão (existência de: algum tipo de supervisão; normas técnicas disponíveis), Estrutura (questões referentes a existência das estruturas necessárias para a condução das atividades em cada nível operacional) e Vacina (questões referentes ao conhecimento sobre a vacina que o Brasil adotou e faixa etária vacina).

Além disso, realizou-se uma análise documental (Plano de Capacitação, Planos de Ação, Normas técnicas, Planilha Orçamentária, Protocolo de Tratamento do Paciente com Rotavírus, Marcos Regulatórios Operacionais) e uma observação direta da rotina de serviço dentro das respectivas unidades $^{(18,25)}$. A avaliação consistiu em classificar os componentes da VER em suas várias dimensões e níveis como adequados ou inadequados, de acordo com os indicadores da matriz de julgamento.

Esta pesquisa recebeu aprovação do Comitê de Ética em Pesquisa da Escola Nacional de Saúde Pública Sergio Arouca - sob número de parecer CEP/ENSP N78/10. 

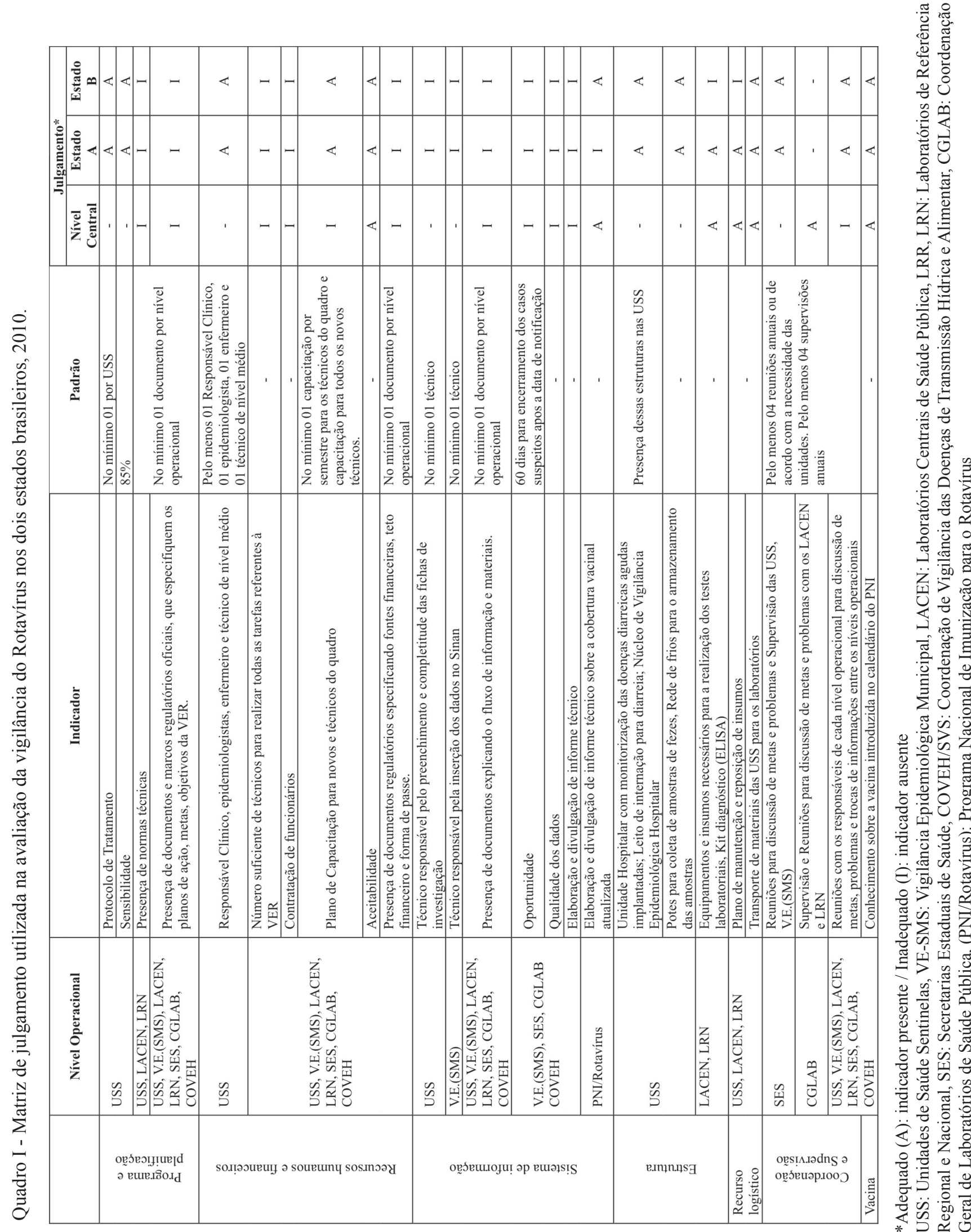


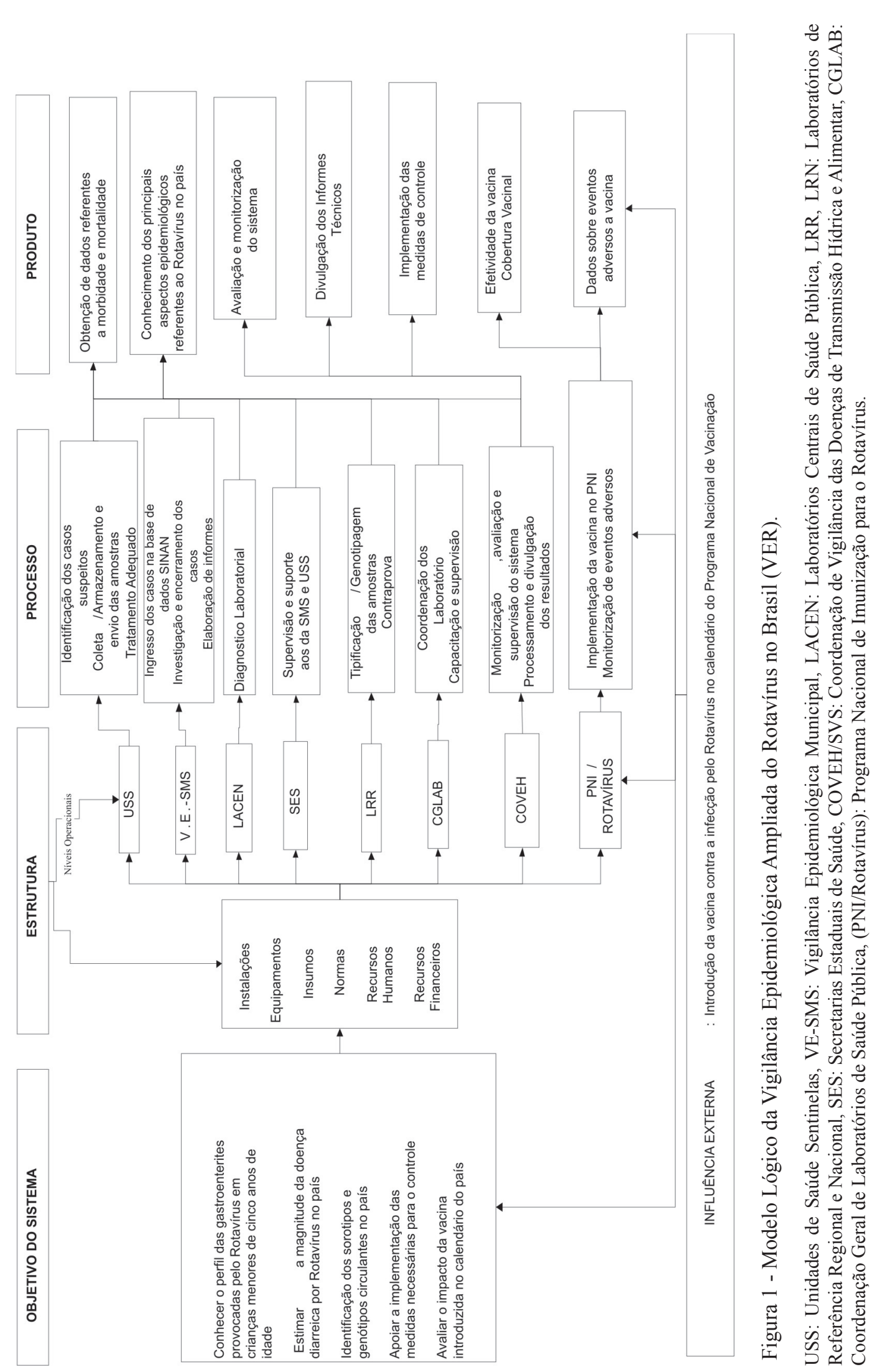




\section{RESULTADOS}

Os dois Estados avaliados não apresentaram o mesmo desempenho operacional evidenciado pela matriz de julgamento (Quadro I). Em todos os níveis, a dimensão Programa e Planificação se apresentou inadequada quanto ao indicador "Presença de documentos e marcos regulatórios oficiais", pela ausência de planos de ação, metas e objetivos. As unidades de saúde em ambos os Estados possuíam normas técnicas operacionais para conduta clínica de casos suspeitos, com sensibilidade para identificar e captar casos, conduzir o tratamento, coletar e armazenar amostras.

Recursos Humanos representou a maior limitação do desempenho da vigilância observada em todos os níveis operacionais. Não houve disponibilização de técnicos, o que ocasionou sobrecarga de funções para os profissionais que desempenhavam outras atividades não relacionadas à VER. No entanto, em cada nível avaliado, havia ao menos um profissional responsável por coordenar e desenvolver as atividades, embora sem capacitação específica.

A dimensão Recursos Financeiros foi avaliada como inadequada em todos os níveis, uma vez que não há a disponibilidade de documentos regulatórios especificando fontes financeiras, teto financeiro e forma de repasse dos recursos, com exceção dos laboratórios. Para os LACEN, os recursos financeiros são oriundos do FINLACEN (Fator de Incentivo aos LACEN-FINLACEN), instituído pela Portaria 2606 do ano de 2005 do Ministério da SaúdeBrasil. Trata-se de um sistema de financiamento que define o repasse de recursos para esses laboratórios de todos os estados do país. O valor do montante repassado é determinado pela classificação dos laboratórios em cinco níveis, que levam em conta a complexidade dos exames que realiza, o seu nível de qualidade e o atendimento de normas de biossegurança e de estabelecimento de um sistema de gestão da qualidade.

O Sistema de Informação mostrou-se inadequado em vários indicadores e níveis operacionais. Exceto na USS do Estado $\mathrm{B}$, não há produção e divulgação de boletins epidemiológicos, deixando diversos níveis operacionais sem retroalimentação. A inserção dos dados no sistema de informação e troca de informações entre os níveis operacionais ainda não ocorre de forma sistemática, prejudicando a completitude dos campos da ficha de investigação; a oportunidade de encerramento dos casos; e a qualidade dos dados de uma forma geral.

A dimensão Estrutura foi avaliada como adequada, nos vários níveis e indicadores. A única exceção foi o LACEN do Estado B, que não dispõe de capacidade instalada para a realização dos testes diagnósticos. O laboratório funciona apenas como um intermediário que armazena as amostras e as envia para o Laboratório de Referência Regional.
Todos os indicadores das dimensões Recurso Logístico, Coordenação e Supervisão e Vacina apresentaram resultados adequados nos níveis operacionais visitados. $\mathrm{O}$ único indicador que teve sua avaliação inadequada foi "Reuniões com os responsáveis de cada nível operacional para discussão de metas, problemas e trocas de informações entre os níveis operacionais". Isto sugere a pouca assistência, orientação e supervisão pelo nível central.

\section{DISCUSSÃO}

A presente pesquisa avaliativa da Vigilância Epidemiológica Ampliada do Rotavírus no Brasil (VER), implementada no país desde 2006, mostrou diversos pontos positivos, dentre o quais se pode destacar: a sensibilidade de captação de casos, a aceitabilidade do sistema por parte dos técnicos envolvidos, estruturas físicas adequadas para a realização das atividades de rotina e capacitação e atualização dos técnicos. No entanto, ficaram evidenciadas também dificuldades operacionais como a ausência de um sistema de informação funcional e oportuno; e a escassez de recursos financeiros e humanos, evidenciando ainda uma fragilidade do sistema.

Uma vigilância epidemiológica capaz de fornecer informações para detectar mudanças nos fatores determinantes e subsidiar recomendações é fundamental para o controle de qualquer agravo. A Vigilância Epidemiológica Ampliada do Rotavírus é, assim, peça chave dentro das atividades desenvolvidas para o monitoramento do perfil do agravo, principalmente após a implementação da vacina no calendário básico de vacinação infantil no Brasil(27).

De acordo com a $\mathrm{OMS}^{(28)}$, a oportunidade e a qualidade dos dados da vigilância do Rotavírus pode fornecer estimativas iniciais de carga da doença, subsidiando assim a adoção de medidas de controle, além de ajudar no monitoramento do impacto da introdução da vacina. As dificuldades operacionais encontradas no presente estudo demonstraram que a VER foi implantada com pouco planejamento, não havendo criação de estruturas diferenciadas, nem alocações de recursos financeiros e humanos para o desenvolvimento das atividades específicas dessa vigilância. Essas limitações estruturais prejudicam o suporte ao processo de informação-decisão-ação, indo contra o princípio de oportunidade e dinamismo das vigilâncias.

Assim, observou-se na atual investigação que a vigilância do Rotavírus em alguns níveis operacionais vem se mostrando incipiente por vários motivos, dentre eles as atividades desenvolvidas nesses níveis estão sendo realizadas em função de outras, não necessariamente ligadas às atividades de rotina da VER, e não há disponibilização de recursos humanos e/ou financeiros próprios para a condução 
das atividades da vigilância em unidades chaves como as unidades sentinelas e os LRR.

Essas condições são desfavoráveis para a consolidação das atividades da VER, uma vez que a dependência de editais de pesquisas para a obtenção de recursos para a contração de técnicos e compra de insumos, deixa as atividades da vigilância extremamente vulneráveis ${ }^{(23,25,26)}$.

Os Sistemas de Informação em Saúde (SIS) podem ser entendidos como ferramentas para a produção de informações que direcionam o processo decisório dos profissionais dos serviços dos diferentes níveis de atenção à saúde. Assim, as informações detectadas em tempo real, auxiliam no planejamento e execução de ações, de acordo com a realidade e especificidade do serviço ${ }^{(29)}$. Dentro desse contexto, o sistema de informação da VER não está funcionando de forma oportuna nos estados avaliados na presente pesquisa, visto que esse é diretamente afetado pela escassez de recursos humanos, considerados como o maior ponto de estrangulamento da vigilância. Os técnicos não conseguem obter indicadores epidemiológicos básicos, e consequentemente, com exceção da USS do Estado B, informes ou boletins epidemiológicos não são elaborados, deixando o sistema sem retroalimentação.

De um modo geral, a troca de informação está sendo realizada através da divulgação de planilhas com os resultados do laboratório de referência regional, e dentro da concepção de vigilância epidemiológica, a divulgação de dados brutos por meio de planilhas não é suficiente para a detecção de perfis do agravo ou mesmo para auxiliar na tomada de decisões ${ }^{(30)}$.

Para a melhoria do sistema de informação é fundamental que níveis operacionais como VE-SMS, SES e COVEH trabalhem os dados e divulguem as informações na forma de boletins epidemiológicos contextualizados por suas regiões ou mesmo comparando as diversas regiões do país. Além disso, busca-se o fortalecimento da relação de troca de informação sistematizada entre os níveis operacionais da VER e o PNI/Rotavírus.

A presente avaliação apresentou limitações, de forma que não foi capaz de obter informações para responder a todos os indicadores propostos pela matriz de julgamento. Indicadores como sensibilidade, oportunidade e qualidade dos dados não puderam ser mensurados nem analisados nessa pesquisa. A avaliação não teve acesso aos bancos de dados para o cálculo e obtenção daqueles indicadores, sendo esta uma limitação do estudo. No entanto, em futuras avaliações, é fundamental essa etapa de análise dos dados, que pode revelar importantes deficiências da vigilância. Além disso, o modelo foi eficiente em detectar a carência de recursos financeiros e humanos nas unidades, porém, inadequado para explicar a lógica de alocação dos recursos e propor recomendações.
Os resultados encontrados na presente pesquisa sugerem a inadequação do tipo de vigilância adotada. Por isso, a busca pela adequação da vigilância do Rotavírus é essencial para a obtenção de dados epidemiológicos que norteiem as políticas de saúde para o controle do agravo. A adoção de uma vigilância epidemiológica do tipo Sindrômica pode ser a mais indicada, por sua robustez e compatíbilidade com a organização e dinâmica do sistema único de saúde brasileiro.

Esse tipo de vigilância pode utilizar diversas formas e fontes de dados, que não são necessariamente casos suspeitos ou confirmados, para detectar de forma precoce a alteração do perfil do agravo ${ }^{(30-32)}$. No caso do Rotavírus, a estruturação da vigilância sindrômica se apoiaria principalmente na captação de casos de diarreias em unidades de media e baixa complexidade e as amostras coletadas seriam enviadas aos laboratórios ${ }^{(30)}$. Como vantagens ter-se-ia que a fonte de informação não estaria concentrada em um único ponto no estado.

Esse aspecto é muito relevante quando se pretende levantar o perfil epidemiológico do agravo, uma vez que amostras serão coletadas em diversos pontos com diferentes características e em populações com diferentes coberturas vacinais. Também seria possível, em um curto período, ampliar para todas as unidades federativas do país esse modelo, já que ele não prescinde de estruturas diferenciadas.

Por fim, a abordagem utilizada no presente estudo envolveu, em todas as suas fases, os atores de diferentes dimensões e níveis operacionais da VER. Assim, a avaliação para os atores deixou de ter um caráter perturbador e punitivo das atividades desenvolvidas ${ }^{(33)}$. Ao contrário, passou a ser percebida como uma importante ferramenta de melhora da qualidade das atividades de rotina ${ }^{(19)}$.

\section{CONCLUSÃO}

Os resultados encontrados sugerem a baixa efetividade do sistema de vigilância adotado, indicando que a vigilância do tipo sentinela pode não ser a mais adequada para o sistema de saúde desses estados estudados.

\section{AGRADECIMENTOS}

À Coordenação de Vigilância das Doenças de Transmissão Hídrica e Alimentar (COVEH/SVS), à Coordenação Geral de Laboratórios de Saúde Pública (CGLAB) e ao Programa Nacional de Imunização para o Rotavírus (PNI/Rotavírus), a todos os técnicos das unidades visitadas e à Escola Nacional de Saúde Pública Sergio Arouca ENSP/FIOCRUZ.

Neste trabalho não há conflitos de interesse entre os autores. 


\section{REFERÊNCIAS}

1. De Oliveira LH, Giglio N, Ciapponi A, Martí SG, Kuperman M, Sanwogou NJ, Ruiz-Matus C, Marinho de Sousa MF. Temporal trends in diarrhea-related hospitalizations and deaths in children under age 5 before and after the introduction of the rotavirus vaccine in four Latin American countries. Vaccine. 2013;31(3):99-108.

2. Sanchez PE, Grais RF, Guerin PJ, Steele AD, Burny ME, Luquero FJ. Burden of disease and circulating serotypes of rotavirus infection in sub-Saharan Africa: systematic review and meta-analysis. Lancet Infect Dis. 2009;9(9):567-76.

3. Parashar UD, Glass RI. Rotavirus Vaccines - Early Success, Remaining Questions. N. Engl. J Med. 2009;360(11):1063-5.

4. Tate JE, Burton AH, Boschi-Pinto C, Steele AD, Duque J, Parashar UD. 2008 estimate of worldwide rotavirus-associated mortality in children younger than 5 years before the introduction of universal rotavirus vaccination programmes: a systematic review and meta-analysis. Lancet Infect Dis.2012;12(2):136-41.

5. Justino MC, Araújo EC, Van Doorn LJ, Oliveira CS, Gabbay YB, Mascarenhas JD, et al. Oral live attenuated human rotavirus vaccine (Rotarix ${ }^{\mathrm{TM}}$ ) offers sustained high protection against severe G9P[8] rotavirus gastroenteritis during the first two years of life in Brazilian children. Mem Inst Oswaldo Cruz. 2012;107(7):846-53 .

6. De la Hoz-Restrepo F, Castañeda-Orjuela C, Paternina A, Alvis-Guzman N. Systematic review of incremental non-vaccine cost estimates used in cost-effectiveness analysis on the introduction of rotavirus and pneumococcal vaccines. Vaccine. 2013;31(3):80-7.

7. Desai R, Oliveira LH, Parashar UD, Lopman B, Tate JE, Patel MM. Reduction in morbidity and mortality from childhood diarrhoeal disease after species A rotavirus vaccine introduction in Latin America: a review. Mem Inst Oswaldo Cruz. 2011;106(8):907-11.

8. Costa MS, Nogueira PA, Magalhães GF, Taquita P, Mariúba LA, Penatti M, et al. Rotavirus genotyping in gastroenteritis cases of an infantile population from Western Brazilian Amazonia. Rev Soc Bras Med Trop. 2012;45(4):520-2.

9. World Health Organization - WHO. Rotavirus vaccines: WHO position paper-January 2013. Wkly Epidemiol Rec. 2013;88(5):49-64.
10. Oliveira LH, Toscano CM, Sanwogou NJ, Ruiz-Matus C, Tambini G, Roses-Periago M, et al. Systematic documentation of new vaccine introduction in selected countries of the Latin American Region. Vaccine. 2013;31(3):114-22.

11. Flannery B, Samad S, Moraes JC, Tate JE, DanovaroHolliday MC, Oliveira LH, Rainey JJ. Uptake of oral rotavirus vaccine and timeliness of routine immunization in Brazil's National Immunization Program. Vaccine. 2013;31(11):1523-8.

12. Organización Panamericana de la Salud. Vigilancia epidemiológica. In: Organización Panamericana de la Salud . Vigilancia epidemiológica de diarreas causadas por rotavirus: Guía práctica. Washington DC; 2007. p. 8-19.

13. Centers for Disease Control and Prevention - CDC. Rotavirus surveillance: worldwide, 2009. Morb Mortal Wkly Rep. 2011;60(16):514-6.

14. Ministério da Saúde (BR). Vigilância do Rotavírus no Brasil. Brasília; 2013.

15. Contandriopoulos AP, Champagne F, Denis JL, Pineault R. A avaliação na área da saúde: conceitos e métodos. In: Hartz ZMA, organizadora. Avaliação em saúde: dos modelos conceituais a práticas da implantação de programas. Rio de Janeiro: Editora Fiocruz; 1997. p. $29-47$.

16. Matida AH, Camacho LAB. Pesquisa avaliativa e epidemiologia: movimentos e síntese no processo de avaliação de programas de saúde. Cad Saúde Pública. 2004;20(1):37-47.

17. Denis JL, Champagne F. Análise de implantação. In: Hartz ZMA, organizadora. Avaliação em saúde: dos modelos conceituais a práticas da implantação de programas. Rio de Janeiro: Editora Fiocruz; 1997. p. 49-88.

18. Yin R. Case study research: Design and methods. 2th ed Beverly Hills, CA: Sage Publishing; 1994.

19. Chen HT. Theory-driven evaluations. Newbury Park: Sage Publications; 1997.

20. McLaughlin JA, Jordan GB. Logic models: A tool for telling your program's performance story. Evaluation and Program Planning. 1999;22(1):65-72.

21. Center for Disease Control and Prevention. Framework for program evaluation in public health. MMWR Recomm Rep. 1999;48(RR-11):1-40. 
22. W.K. Kellogg Foundation. Logic model development guide. [cited 2004]. Available from: www.wkkf.org/ Pubs/Tools/Evaluation/Pub3669.pdf.

23. Hartz ZMA, Silva LMV. Avaliação em saúde: dos modelos teóricos à prática na avaliação de programas e sistemas de saúde. Salvador: EDUFBA/Rio de Janeiro: Editora Fiocruz; 2005.

24. Helitzer D, Hollis C, Hernandez BU de, Sanders M, Roybal S, Van Deusen I. Evaluation for communitybased programs: The integration of logic models and factor analysis. Evaluation and Program Planning. 2010;33(3):223-33.

25. Patton M. Essentials of utilization-focused evaluation. Thousand Oaks, Ca: Sage; 2012.

26. Poon B, Leung JW, Louie A, Vergel de Dios C. The key functions of collaborative logic modeling: Insights from the British Columbia Early Childhood Dental Programs. Canadian J Program Evaluation. 2013;27(2):87-102.

27. Patel MM, Glass R, Desai R, Tate JE, Parashar UD. Fulfilling the promise of rotavirus vaccines: how far have we come since licensure? Lancet Infect Dis. 2012;12(7):561-70.

28. World Health Organization. Building rotavirus laboratory capacity to support the Global Rotavirus Surveillance Network. Wkly Epidemiol Rec. 2013;88(21):217-24.
29. Cavalcante RB, Brito MJM, Porto F. Sistema de Informação: contribuições e desafios para o cotidiano de trabalho em unidades de terapia intensiva de Belo Horizonte. J. Health Inform. 2009;1(1):34-42.

30. Van den Wijngaard CC, Van Pelt W, Nagelkerke NJ, Kretzschmar M, Koopmans MP. Evaluation of syndromic surveillance in the Netherlands: its added value and recommendations for implementation. Euro Surveill. 2011;16(9):1-8.

31. O’Connell EK, Zhang G, Leguen F, Llau A, Rico E. Innovative uses for syndromic surveillance. Emerg Infect Dis. 2010;16(4):669-71.

32. Heffernan R, Mostashari F, Das D, Karpati A, Kulldorff M, Weiss D. Syndromic Surveillance in Public Health Practice, New York City. Emerg Infect Dis. 2004;10(5):858-864.

33. Bryson, JM, Patton MQ, Bowman RA. Working with Evaluation Stakeholders: A Rationale, StepWise Approach and Toolkit. Eval Program Plann. 2011;34(1):1-12.

\section{Endereço para correspondência:}

Robson Bruniera de Oliveira

Rua Gastão Madeira, 26

Bairro: Praia da Toninhas

CEP: 11680-00 - Ubatuba - SP - Brasil

E-mail: robsonb@fiocruz.br 